\title{
A Geometric Theory of Chaotic Phase Synchronization
}

\author{
Margaret Beck and Krešimir Josić* \\ Department of Mathematics and Statistics and Center for BioDynamics, \\ Boston University, Boston, MA 02215
}

February 19, 2003

\begin{abstract}
A rigorous mathematical treatment of chaotic phase synchronization is still lacking, although it has been observed in many numerical and experimental studies. This article addresses the extension of results on phase synchronization in periodic oscillators to systems with phase coherent chaotic attractors with small phase diffusion. As models of such systems we consider special flows over diffeomorphisms in which the neutral direction is periodically perturbed. A generalization of the Averaging Theorem for periodic systems is used to extend Kuramoto's geometric theory of phase locking in periodically forced limit cycle oscillators to this class of systems. This approach results in reduced equations describing the dynamics of the phase difference between drive and response systems over long time intervals. The reduced equations are used to illustrate how the structure of a chaotic attractor is important in its response to a periodic perturbation, and to conclude that chaotic phase coherent systems may not always be treated as noisy periodic oscillators in this context. Although this approach is strictly justified for periodic perturbations affecting only the phase variable of a chaotic oscillator, we argue that these ideas are applicable much more generally.
\end{abstract}

\footnotetext{
*josic@math.bu.edu
} 
The theory of phase synchronization between interacting periodic oscillators plays a central role in the mathematical analysis of pattern formation, design of phase lock loops, mathematical neuroscience, and a number of other areas of physics, engineering and applied mathematics. A number of experimental and numerical studies have recently demonstrated that even chaotic oscillators can display phase locking. In this article we extend the theory of phase locking developed for periodically perturbed limit cycle oscillators to a class of periodically driven chaotic oscillators which serve as a model for a wide class of phase coherent systems. In this case the equations modeling the phase difference between the periodic perturbation and the oscillator take the form of a circle map driven by a diffeomorphism obtained from a Poincaré section of the chaotic attractor. These equations can lead to very complicated behavior since the structure of the original chaotic attractor plays an important role in their derivation. We illustrate some of the possibilities for the long term behavior of the phase difference, including examples in which the correlation between the drive and response signals decreases with an increase in coupling strength, contrary to intuition. We demonstrate that the phase difference in periodically perturbed chaotic systems may display a range of behaviors that may not always be adequately captured by approximating the chaotic oscillator with a stochastically perturbed periodic oscillator.

\section{Introduction}

The phenomenon of phase synchronization between periodically oscillating systems, first noted by Huygens [1], has been extensively studied and is well understood. The recent observation of the phenomenon in chaotic systems $[2,3,4]$ has prompted a large number of numerical and experimental studies. Chaotic phase synchronization (CPS) has since been detected in studies of electrically coupled neurons $[5,6]$, spatially extended ecological systems [7], earthquake models [8], and a periodically driven plasma discharge tube [9], to cite just a few in a growing number of examples. However, there are few rigorous results about CPS in the literature, and several of its features are still not well understood.

Several approaches have been used to give a heuristic explanation of CPS and to describe some of its aspects. In [4] it is shown that it is frequently possible to define a phase variable which is periodic up to a small chaotic term. For systems in which this chaotic term can be treated as white noise, the methods introduced by Stratonovich work well [10]. Another approach is to study the phase locking properties of the 
entire attractor in terms of the behavior of periodic orbits embedded in it [11]. Since the periodic orbits form a skeleton of the attractor, this approach is natural. Other interesting properties of chaotic phase locked attractors were studied in [12, 13].

Such approaches provide effective ways of studying CPS, however they are frequently difficult to justify rigorously. There are also several fundamental questions which are hard to address using the ideas proposed so far: What type of chaotic systems can display CPS? Is it possible to directly extend the well developed theory of phase locking of periodic oscillators to the chaotic case? If so, can chaotic oscillators in general be thought of as noisy periodic oscillators?

In this paper we develop a rigorous approach to the study of CPS in a somewhat idealized model which gives a partial answer to these questions. There are several ways to study phase locking of periodic systems [14, 15, 16, 17]. We will combine the idea of reducing a phase coherent system to a special flow over a diffeomorphism [11], with the geometric description of periodic phase locking given by Kuramoto [17] to provide both a geometrical and mathematical explanation of when and how CPS occurs. On the basis of this description we develop a model and use it to show that we can typically expect two obstacles to phase synchronization: one due to the phase diffusion on the attractor and the second due to the variation of the sensitivity of the phase across the attractor. In the case of a periodically driven chaotic system the first obstacle can be eliminated by an increase in the coupling strength. However, this is not true for the second obstacle. This allows us to conclude that chaotic oscillators cannot in general be treated as noisy periodic oscillators for the purpose of studying their phase locking properties. Although we only address the problem of a chaotic system subject to a periodic perturbation, a similar theory can be developed for coupled phase coherent systems.

We start by reviewing Kuramoto's analysis of phase locking in periodic systems in Section 2, and show how to extend this approach to chaotic systems in Section 3. In Section 4 we introduce an idealized model of a periodically driven chaotic attractor. We next use an extension of the standard Averaging Theorem to find a simplified equation for the dynamics of the phase difference in Section 5. In certain situations a further simplification is possible by averaging over the invariant measure on the attractor. However if the convergence to the averages is insufficiently fast, this reduction may not be possible on parts of the attractor. These issues and their effect on the phase locking properties of the system are discussed in Sections 6 and 7. The proofs of the results used in the paper are contained in the Appendix. 


\section{Phase locking in periodic systems}

We first review the basic principles behind the analysis of phase locking of periodic oscillators because they form the basis of our approach to the analysis of chaotic phase synchronization. Suppose that a system $y^{\prime}=f(y)$ with $y \in \mathbb{R}^{n}$ has an exponentially stable periodic orbit of period $T$. Consider the perturbed system $y^{\prime}=f(y)+\epsilon s(t)$ where $\epsilon s(t)$ is a small $T^{\prime}$-periodic signal and $T$ and $T^{\prime}$ are assumed to be close. Let $\sim$ denote the identification of the endpoints of the interval $[0, T]$ so that $[0, T] / \sim=S^{1}$. It is shown in $[15,17]$ that there exists a phase coordinate $\phi(y) \in$ $[0, T] / \sim$ defined in the neighborhood of the unperturbed limit cycle representing the phase of the driven system. Under the periodic perturbation $\epsilon s(t)$ the evolution of the phase $\phi$ is governed by

$$
\phi^{\prime}=1+\epsilon \nabla \phi \cdot s(t) \stackrel{\text { def }}{=} 1+\epsilon G(t, \phi)
$$

where $G(t, \phi)=\nabla \phi \cdot s(t)$. The gradient of $\phi(y)$ measures the sensitivity of the phase to perturbations, and is therefore called the phase dependent sensitivity. Since the signal $s(t)$ is $T^{\prime}$-periodic we may think of $\phi_{d}=\left(T / T^{\prime}\right) t \bmod T$ as the phase of the driving signal, so that $\phi_{d} \in[0, T] / \sim$. Therefore the phase difference $\Psi=\phi-\phi_{d}$ can also be written as

$$
\Psi=\phi-\frac{T}{T^{\prime}} t \quad \bmod T
$$

and it satisfies

$$
\Psi^{\prime}=\Delta+\epsilon G\left(t, \Psi+\frac{T}{T^{\prime}} t\right)
$$

where $\Delta=1-\frac{T}{T^{\prime}}$.

$G\left(t, \Psi+\frac{T}{T^{\prime}} t\right)$ is $T^{\prime}$-periodic in t, and since $T-T^{\prime}$ is small we can assume that $\Delta=\mathcal{O}(\epsilon)$. Thus averaging is justified and we obtain the reduced equation

$$
\Psi^{\prime}=\Delta+\epsilon \Gamma(\Psi)
$$

where $\Gamma(\Psi)=1 / T^{\prime} \int_{0}^{T^{\prime}} G\left(t, \Psi+\frac{T}{T^{\prime}} t\right) d t$. Averaging guarantees that the solutions of this equation are close to the actual solution up to and including times of $\mathcal{O}(1 / \epsilon)$. If (3) has a stable fixed point, then the original equation has a $T^{\prime}$-periodic solution in an $\mathcal{O}(\epsilon)$ neighborhood of this fixed point so that the phase difference $\phi-\phi_{d}$ is nearly fixed and the oscillator is phase locked with the drive. 


\section{Extensions to chaotic systems}

The concept of phase locking can be extended to a system $y^{\prime}=f(y), y \in \mathbb{R}^{n}$ and $f$ smooth, with a chaotic attractor $A$ on which a meaningful phase can be defined. Such systems are typically called phase coherent, and are a natural generalization of periodically oscillating systems. We define phase coherence as follows

Definition 3.1 An attractor $A$ is phase coherent if there exists a smooth, transversal section $\Sigma$ of the attractor and constants $T>0$ and $\epsilon>0$ such that all orbits starting at $\Sigma$ return to $\Sigma$ within a time $t$ where $|T-t|<\epsilon$.

It is natural to extend this definition to systems for which the conditions hold for $\mu$-almost all, or a large measure of initial conditions on $\Sigma$, where $\mu$ is some natural measure on $\Sigma$ invariant under the Poincaré return map. The constant $\epsilon$ gives a rough measure of how much the flow on $A$ deviates from being periodic. However, $A$ does not have to be periodic or quasiperiodic even when $\epsilon=0$.

To avoid notational complications which would occur as a consequence of defining several charts on the section $\Sigma$, we assume that a single coordinate chart $x: \Sigma \rightarrow$ $\mathbb{R}^{n-1}$ is sufficient. We can therefore think of $\Sigma$ as a subset of Euclidean space $\mathbb{R}^{n-1}$. By a slight abuse of notion we will denote initial conditions on $\Sigma$ by their coordinate $x$. Let $F: \Sigma \rightarrow \Sigma$ be the Poincaré return map induced by the flow, and $T(x)$ the time for a point $x \in \Sigma$ to return to $\Sigma$ under the flow.

In general the phase of a system is a function $\phi: N \rightarrow S^{1}$ from a neighborhood $N$ of the attractor $A$ to the circle. Following Definition 3.1 we consider the circle $S^{1}=[0, T] / \sim$ where $\sim$ denotes identification of 0 and $T$. The following proposition shows that a natural, approximately uniformly increasing phase variable can be defined on a phase coherent attractor.

Proposition 3.2 There exist functions $R: N \rightarrow \mathbb{R}^{n-1}$ and $\phi: N \rightarrow S^{1}$ on a neighborhood $N$ of a phase coherent attractor $A$ such that $(R, \phi)$ are new coordinates on $N$ in which the equations of motion take the form

$$
\begin{aligned}
R^{\prime} & =f(R, \phi) \\
\phi^{\prime} & =1+\delta(R, \phi) .
\end{aligned}
$$

The function $\delta$ can be chosen arbitrarily close to $\frac{T}{T(x)}-1$.

The proof of this Proposition may be found in the Appendix. It follows that the uniform flow on the circle is nearly a factor map of a phase coherent system if $\delta$ is small. Therefore we can expect that the time series of certain coordinates $v: N \rightarrow \mathbb{R}$ will have a peak in their power spectrum, and that the width of this 
peak is related to the distribution of return times to the section $\Sigma$, as already noted in $[4,18]$. For instance if we think of $\phi \in S^{1} \subset \mathbb{R}^{2}$ as moving on a circle in the plane then the time series of the $x$-coordinate of a point on the circle will be nearly sinusoidal.

It is clear from the proof of the Proposition that the coordinates $R$ and $\phi$ are not uniquely defined. However, if $\phi_{1}$ and $\phi_{2}$ are two definitions of the phase obtained from the section $\Sigma$ so that $\phi_{1}^{\prime}=1+\delta_{1}(R, \phi)$ and $\phi_{2}^{\prime}=1+\delta_{2}(R, \phi)$ then

$$
\phi_{1}(y(t, x))-\phi_{2}(y(t, x))=\int_{0}^{t}\left(\delta_{1}\left(R_{1}, \phi_{1}\right)-\delta_{1}\left(R_{1}, \phi_{1}\right)\right) d t .
$$

Therefore $\left|\phi_{1}(y)-\phi_{2}(y)\right|<2 T \max \left(\left|\delta_{1}\right|,\left|\delta_{2}\right|\right)$. If the original system is phase coherent than $\left|\delta_{1}\right|$ and $\left|\delta_{2}\right|$ can be chosen to be small, so that the definitions of the phase are nearly the same. Since only a limit cycle can be parameterized by a single phase variable, it can in general not be expected that $\delta(R, \phi)=0$, however Proposition 3.2 implies that for phase coherent attractors $\phi$ may be defined so that $|\delta(R, \phi)|$ is close to $\left|\frac{T}{T(x)}-1\right|<\epsilon+\mathcal{O}\left(\epsilon^{2}\right)$.

If the original system is subject to a small periodic perturbation $\epsilon s(t)$ of period $T^{\prime}$ so that $y^{\prime}=f(y)+\epsilon s(t)$, we can follow the calculations of Section 2 to obtain

$$
\phi^{\prime}=1+\delta(R, \phi)+\left.\epsilon \nabla_{y} \phi\right|_{(R, \phi)} \cdot s(t)
$$

where $\left.\nabla_{y} \phi\right|_{(R, \phi)}$ is the gradient of $\phi(y)$ at a point $(R, \phi)$.

If we define the phase difference $\Psi$ as in (1), and let $\Delta=1-\frac{T}{T^{\prime}}$ we obtain

$$
\Psi^{\prime}=\Delta+\epsilon G\left(\Psi+\frac{T}{T^{\prime}} t, R\right)+\delta(\Psi, R) .
$$

There are two differences between equations (2) and (7): the term $\delta$ and the dependence of $G$ on $R$ in equation (7). If the autocorrelations of $y$ decay relatively quickly in time it can be expected that $\delta$ acts as a random perturbation since $\Psi$ evolves on a slow time scale compared to $\phi$ and $R$. Moreover, this term is not proportional to the coupling strength $\epsilon$ and it can hence be dominated by the term $\epsilon G$ for sufficiently large coupling values if the system does not undergo bifurcations that significantly change its behavior. These arguments have been used to study chaotic phase synchronization in [4] using the theory of phase synchronization of stochastic oscillators [10].

On the other hand the term $\epsilon G\left(\Psi+\frac{T}{T^{\prime}} t, R\right)$ in (7) is proportional to the coupling strength $\epsilon$. Since the behavior of $R$ and $\phi$ are not necessarily correlated, the influence of this term on the dynamics of $\Psi$ is highly dependent on the particular system. Sometimes the dependence on $R$ is weak, so that $\epsilon G\left(\Psi+\frac{T}{T^{\prime}}, R\right) \approx \epsilon \tilde{G}\left(\Psi+\frac{T}{T^{\prime}}\right)$. 
In such cases the system may frequently be viewed as a stochastically perturbed periodic system $[11,4]$. If the dependence of $G$ on $R$ is strong, the situation is more complicated. Unlike the term $\delta$, an increase in coupling strength will only increase the effect of this term on the dynamics of the phase difference $\Psi$. Examples in [19] show that this may significantly affect the phase locking properties of the attractor, and in the following we explore this situation further.

\section{A model system}

In this Section we describe a somewhat simplified model that illustrates how the dependence of the function $G$ on $R$ may influence the phase locking properties of a system. Some of the ideas we present have already been suggested in [11] in a somewhat different context and we make certain steps in this approach rigorous.

Following Definition 3.1 we again assume that there exists a transversal section $\Sigma$ of the attractor of a system $y^{\prime}=f(y)$. This section corresponds to the constant phase $\phi=0$ in a neighborhood of the attractor. Let $F(x)$ be the Poincaré map on $\Sigma$ and note that by smooth dependence on initial conditions the return time $T(x)$ of a point $x \in \Sigma$ to $\Sigma$ is as smooth as the flow $\varphi^{t}$.

Next we define a special flow $g^{t}$ over the Poincaré map $F[20]$. Let $Y=\{(x, \phi) \in$ $\Sigma \times \mathbb{R}: 0 \leq \phi<T(x)\}$. The flow is defined for for positive $t$ and $\phi \in[0, T(x))$ by

$$
\begin{aligned}
& g^{t}(x, \phi)=(x, \phi+t) \quad \text { if } 0 \leq \phi+t<T(x) \\
& g^{t}(x, \phi)=\left(F^{k}(x), u\right) \quad \text { if } 0 \leq u=t+\phi-\sum_{0 \leq i \leq k-1} T\left(F^{i}(x)\right)<T\left(F^{k}(x)\right)
\end{aligned}
$$

where $k=\max \left\{i \geq 1: \phi+t-\sum_{j=0}^{i} T\left(F^{j}(x)\right) \geq 0\right\}$. The flow can be defined similarly for negative $t$ for points which are on the attractor $A$. See Fig. 1.

Standard arguments show that the manifold $\tilde{Y}=Y / \sim$ obtained by identifying $(x, T(x))$ and $(F(x), 0)$ can be given a structure of a smooth manifold with boundary and that the flow $g^{t}$ generates a flow on this manifold. We can define a function $h: N \rightarrow \tilde{Y}$ on some neighborhood $N$ of $A$ by

$$
h\left(\varphi^{t}(x, 0)\right)=\left(F^{k}(x), u\right)
$$

with $k$ as above and $u=t-\sum_{0<i<k-1} T\left(F^{i}(x)\right)<T\left(F^{k}(x)\right)$. It is easy to check that $h$ can be used to define a smooth conjugacy between the flows $\varphi^{t}$ and $g^{t}$ on their respective attractors. This conjugacy can be extended to a neighborhood of the attractors for positive time.

Note that $h$ preserves time which will be important in our considerations. Therefore the flows $\varphi^{t}$ and $g^{t}$ are equivalent in a positively invariant neighborhood of 
their attractors with the equivalence preserving parameterization in time, and we may think of $(x, \phi)$ as new coordinates in the neighborhood of the attractor. Since $\phi$ changes between 0 and $T(x)$ during one oscillation it is more convenient to think of the quantities $\frac{T}{T(x)} \phi$ as the phase on the attractor of this system so that we may think of the phase as defined on $[0, T] / \sim=S^{1}$.

Assume that $F: \Sigma \rightarrow \Sigma$ preserves some natural invariant measure $\mu$ on $\Sigma$. We let $\langle\cdot\rangle_{\mu}=\langle\cdot\rangle$ denote the average with respect to this measure. Then $T=\langle T(x)\rangle=$ $\lim _{n \rightarrow \infty} 1 / n \sum_{i=0}^{n-1} T\left(F^{i}(x)\right)$ is the average return time to the section $\Sigma$, and the second equality holds $\mu$-almost everywhere. It is natural to use this $T$ in Definition 3.1 .

Define the stroboscopic map $\phi_{N}=\phi(N T)$, so that $\left\langle\phi_{N}\right\rangle=0$ by the definition of $T$. We will consider the variance of the distribution of $\phi_{N}$ around 0 . The rate of growth of this variance is called the phase diffusion constant. It measures the speed of growth of the distribution of phases in an ensemble of systems with equal initial phases, but different initial radii. Given an initial condition $x \in \Sigma$, the distance of the point $\phi_{N}$ from $\Sigma$ is given by $\sum_{n=0}^{N-1} T\left(F^{n}(x)\right)-N T$, so that phase diffusion constant can be defined as the rate of growth of

$$
V(N)=\left\langle\left(\sum_{n=0}^{N-1}\left(T\left(F^{n}(x)\right)\right)-N T\right)^{2}\right\rangle
$$

or similarly $D_{\phi}=\lim _{n \rightarrow \infty} V(N) / N[21]$. It can be shown that the central limit theorem holds for uniformly hyperbolic dynamical systems $[22,23]$, so that if $F$ is uniformly hyperbolic

$$
\frac{V(N)}{N} \rightarrow\left\langle\left((T(x))^{2}\right\rangle-T^{2}+2 \sum_{n=1}^{\infty}\left\langle T\left(F^{n}(x)\right)-T\right\rangle\langle T(x)-T\rangle .\right.
$$

This result may be expected to hold in the case of many physical systems [24].

This quantity provides a good measure of how much the return times $T(x)$ deviate from the average return time $T$. If system (8) can be treated as a stochastically perturbed periodic system the results in $[10,4]$ suggest that this quantity determines how difficult it is to phase lock this system as compared to the unperturbed periodic system. We will see that in general more information about the structure of the attractor will be needed.

We now assume that the phase $\phi$, and only the phase, is subject to a small, additive perturbation of period $T^{\prime}=T+\mathcal{O}(\epsilon)$. The assumption that the perturbation acts only on the phase is a simplification. Such couplings are realistic in certain systems such as Josephson junctions and phase lock loops [25, 26] and have been considered in the context of chaotic phase synchronization in $[27,11]$. If the 


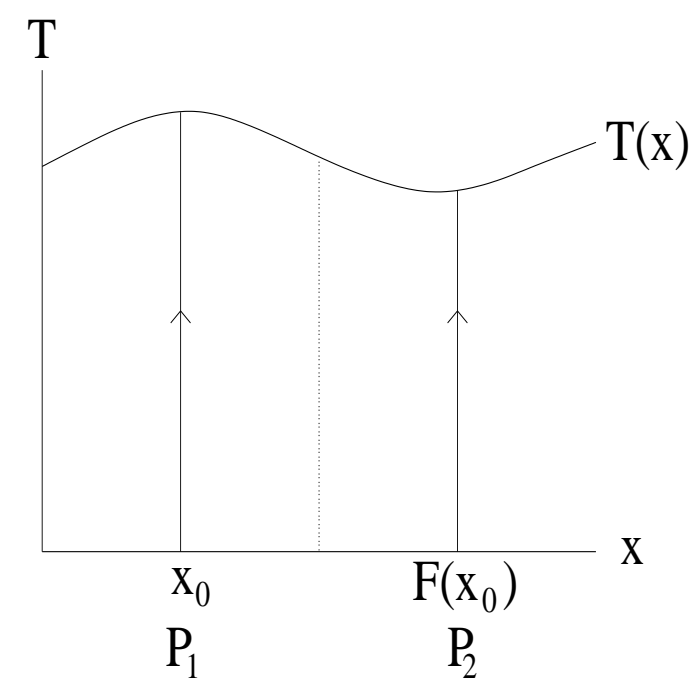

Figure 1: The special flow over a map $F(x)$. The initial point $\left(x_{0}, 0\right)$ gets reinjected at $\left(F\left(x_{0}, 0\right)\right.$ after reaching $\left(x_{0}, T\left(x_{0}\right)\right)$. The average return time is the average of the function $T(x)$. We will use a partition of the Poincaré section $\Sigma$ in the examples in Sections 6 and 7.

perturbation also influences the dynamics of $x$, the situation is more difficult to treat, however if the attractor of the map $F$ is uniformly hyperbolic most of the results below can be expected to carry over directly for sufficiently small values of $\epsilon$ $[28,29]$. This situation is not typical, and in the case of non-uniformly hyperbolic systems even small perturbations can lead to bifurcations and drastic changes in the dynamics of $x$. Since our results are derived by perturbing from $\epsilon=0$, they may no longer be valid in this case, although results derived for hyperbolic systems frequently seem to apply approximately to many non-uniformly hyperbolic systems.

For initial conditions $\left(x_{0}, 0\right)$, let $S_{-1}^{0}=0$ and $S_{n}^{0}=\sum_{0 \leq i \leq n-1} T\left(F^{i}(x)\right)$, so that $S_{n}^{0}$ is the total time to the $n$-th return of the point $\left(x_{0}, 0\right)$ to the section $\Sigma$. Following Section 3 the dynamics of system (8) under a small periodic perturbation is given by

$$
\begin{array}{rlr}
\phi^{\prime} & =1+\epsilon p\left(t, \phi, x_{n}\right) & \\
x(t) & =x_{n} & \text { for } S_{n-1}^{0} \leq \tilde{\phi}<S_{n}^{0}
\end{array}
$$

where $p\left(t, \phi, x_{n}\right)$ is $T^{\prime}$-periodic in $t$, and $\tilde{\phi}$ is the lift of $\phi$ to $\mathbb{R}$. Because of the relation between the special flow on $\tilde{Y}$ and the original system we can assume that $p(t, 0, F(x))=p(t, T(x), x)$, and that $p$ is smooth in all variables. We can obtain equivalent equations for $\tilde{\phi}$, the lift of $\phi$ to the line. 
If we think of $\frac{T}{T^{\prime}} t \bmod T$ as the phase of the driving signal, and $\frac{T}{T(x)} \phi$ as the phase of the system, the phase difference $\Psi \in[0, T)$ between the two can be defined as in (1)

$$
\Psi=\frac{T}{T(x)} \phi-\frac{T}{T^{\prime}} t \quad \bmod T
$$

The phase difference satisfies the equation

$$
\begin{aligned}
\Psi^{\prime} & =\left(\frac{T}{T\left(x_{n}\right)}-\frac{T}{T^{\prime}}\right)+\epsilon \frac{T}{T\left(x_{n}\right)} p\left(t, \phi, x_{n}\right) & & \text { for } S_{n-1}^{0} \leq \tilde{\phi}<S_{n}^{0} \\
& =\Delta\left(x_{n}\right)+\frac{T}{T\left(x_{n}\right)} \epsilon p\left(t, \frac{T\left(x_{n}\right)}{T} \Psi+\frac{T(x)}{T^{\prime}} t, x_{n}\right) & & \text { for } S_{n-1}^{0} \leq \tilde{\phi}<S_{n}^{0}
\end{aligned}
$$

where $\Delta\left(x_{n}\right)=\frac{T}{T\left(x_{n}\right)}-\frac{T}{T^{\prime}}=\mathcal{O}(\epsilon)$. The dependence on $\tilde{\phi}$ can be eliminated by considering the lift $\tilde{\Psi}$ of $\Psi$ to the line. Since the change of coordinates from $\phi$ to $\Psi$ has a discontinuous first derivative on the section $\Sigma$, the vector field in (11) is discontinuous. These discontinuities occur on a discrete set of points. Since the vector field is well behaved in their vicinity we can obtain solutions of system (11) by integrating between the returns to $\Sigma$ and using the final value of $\Psi$ during one oscillation as the initial value in the integration at the next oscillation. Under the change of coordinates (10) these solutions will agree with those of the original problem, so that the discontinuity in the vector field will pose no problem in the following.

Because the dynamics of $\Psi$ depends on $x_{n}$, these equations will in general not have fixed points or attracting periodic orbits. However, if $|\Psi(t)-\theta|<C$, for some $\theta \in[0, T)$, then at any time $n T^{\prime}$ we have

$$
\left|\frac{T}{T(x)} \phi-\theta\right|<C
$$

so that if $\mathrm{C}$ is sufficiently small, the driven system is phaselocked with the drive with approximate phase difference $\theta$. Since $\frac{T}{T(x)}=1+\mathcal{O}(\epsilon)$, the constant $C$ measures how much the phase can deviate from $\theta$ at the times $n T^{\prime}$, in other words how "tightly" the two systems are phase locked.

\section{Reduction of the phase difference equation}

Since $G$ is not periodic in time, it is not possible to average equation (11) directly as in the periodic case. It is possible to use a moving time average [30], however this approach leads to complications. Instead the equation can be averaged between 
returns to the section $\Sigma$, i.e. over intervals defined by the condition $S_{n-1}^{0} \leq \tilde{\phi}<S_{n}^{0}$. The following theorem shows that such averages can be further simplified and that the averaged system stays close to the original system up to times of order $1 / \epsilon$.

We will consider the Poincaré map of system (11) on the section $\Sigma$. More precisely, if $S_{n}^{\epsilon}$ is the time at which the phase variable $\phi$ crosses $\Sigma$ the $n$-th time, we find a dynamical systems whose orbits remain close to the phase difference $\Psi\left(S_{n}^{\epsilon}\right)$ for times of order $1 / \epsilon$.

Theorem 5.1 If $\Delta\left(x_{n}\right)=\mathcal{O}(\epsilon)$, then the orbits of the Poincaré map on the section $\Sigma$ of the system (11) are $\mathcal{O}(\epsilon)$ close to the orbits of the averaged discrete time system

$$
\begin{aligned}
& \rho_{n+1}=\rho_{n}+\eta\left(x_{n}\right)+\epsilon \Gamma\left(\rho_{n}, x_{n}\right) \\
& x_{n+1}=F\left(x_{n}\right)
\end{aligned}
$$

where $\eta\left(x_{n}\right)=\Delta\left(x_{n}\right) T^{\prime}$ and

$$
\Gamma\left(\rho, x_{n}\right)=\int_{0}^{T^{\prime}} p\left(-\frac{T^{\prime}}{T} \rho+t, \frac{T\left(x_{n}\right)}{T^{\prime}} t, x_{n}\right) d t .
$$

The proof of this theorem and the following proposition may be found in the Appendix. Under certain stability conditions we can extend these results to longer time intervals. If the system (13) has a trapping region so that $\left|\rho_{n}-\theta\right|<C<T$ on an arbitrarily long time interval (including infinite time intervals) then we can conclude that $|\Psi(t)-\theta|<C+\mathcal{O}(\epsilon)$ on the same time interval if the phase diffusion and coupling strength are sufficiently small. This means that for long times $\rho_{n}$ and $\Psi\left(S_{n}^{\epsilon}\right)$ remain in subsets of $[0, T)$ that are $\epsilon$ close, even if $\rho_{n}$ and $\Psi\left(S_{n}^{\epsilon}\right)$ themselves may remain close only for times of order $1 / \epsilon$.

Proposition 5.2 Suppose that $I_{2} \subset I_{1}$ are two subintervals of $[0, T] / \sim=S^{1}$ such that $I_{1}$ and $I_{2}$ are absorbing intervals for system (13) under a particular orbit of $x_{n+1}=F\left(x_{n}\right)$. Then for sufficiently small $\epsilon$, the orbits of the unaveraged system (11) remain in an $\epsilon$ neighborhood of $I_{1}$.

In general it may not be sufficient to require that system (13) has only an absorbing region $I_{2}$ since the orbits starting at the vicinity of that region may visit much of $S^{1}$ before reaching $I_{2}$, and hence the last part of the proof would not go through. However, such examples seem atypical. We also note that it is easy to show that if for a given orbit of $x_{n}=F\left(x_{n-1}\right)$, the phase difference $\rho$ in (13) undergoes phase slips on time scales of order $1 / \epsilon$ the same is true for the original system. In general however, system (13) may not give information about the behavior of the phase difference $\Psi$ for very long times. 
Remark 5.3 We note that the results in this Section apply to single orbits of the system $x_{n}=F\left(x_{n-1}\right)$. It is possible, and typically likely, that the dynamics of $\rho$ will be different for different initial values $x_{0} \in \Sigma$. As noted in [11] in the case $\Gamma$ does not depend on $x_{n}$, some periodic orbits in the attractor may be phase locked while others are not, and it is reasonable to study the phase locking properties of the attractor by examining the proportion of phase locked orbits inside it. This situation will be discussed further in Sections 6 and $\%$.

Rather than try to develop a comprehensive theory of the behavior of system (13) we instead study several examples that illustrate the types of behavior that can be expected in more general systems. We first discuss systems which can be reduced further by averaging over the invariant measure on $\Sigma$, and subsequently consider examples of systems for which such averaging is not appropriate.

Systems of the form (13) have already been used to study phase locking of chaotic systems, however it was assumed that only $\eta$ depends on $x_{n}$ while $\Gamma$ does not. In the following we illustrate what can be expected when both $\Gamma$ and $\eta$ depend on $x_{n}$.

\section{Averaging over an invariant measure on $\Sigma$}

In equation (13) $\Gamma$ can generally be expected to vary smoothly with $x_{n}$. However certain attractors, like the ones found in the Lorenz, Chua and Rikitake systems consist of several parts on which the dynamics is relatively uniform. For certain parameter values these systems can also be considered phase coherent. Motivated by these examples we make the following simplifying assumption on $\Gamma\left(\rho_{n}, x_{n}\right)$ and $\eta\left(x_{n}\right)$. Let $\left\{P_{i}\right\}_{i=1}^{n}$ be a finite partition of the section $\Sigma$ and assume that

$$
\begin{aligned}
\Gamma\left(\rho_{n}, x_{n}\right) & =\Gamma_{i}\left(\rho_{n}\right) & & \text { if } x_{n} \in P_{i} \\
\eta\left(x_{n}\right) & =\eta_{i} & & \text { if } x_{n} \in P_{i}
\end{aligned}
$$

so that $\Gamma$ and $\eta$ achieve only finitely many values for a given $x$ (see Fig. 1). Although condition (15) may be expected to hold only approximately in realistic cases, systems that satisfy this condition are more easily studied and can hence be used to provide transparent examples of phenomena that may be expected to occur generally. Note that since $A \cap \Sigma$ can be separated by open sets in our motivating examples condition

(15) does not imply that the original vector field was discontinuous.

Since the dynamics of $\rho$ is much slower than the dynamics of $x$ it is natural to 
assume that we can average $\Gamma$ and $\eta$ as follows:

$$
\begin{aligned}
\bar{\eta} & =\lim _{N \rightarrow \infty} \frac{1}{N} \sum_{n=0}^{N-1} \eta\left(x_{n}\right)=\int \eta(x) d \mu \\
\bar{\Gamma}(\rho) & =\lim _{N \rightarrow \infty} \frac{1}{N} \sum_{n=0}^{N-1} \Gamma\left(\rho, x_{n}\right)=\int \Gamma(\rho, x) d \mu(x)
\end{aligned}
$$

These averages exist almost everywhere for an ergodic, $F$-invariant probability measure $\mu$. We may therefore expect that the dynamics of system (13) may be further reduced to

$$
\rho_{n+1}=\rho_{n}+\bar{\eta}+\epsilon \bar{\Gamma}\left(\rho_{n}\right)
$$

for almost all initial points. This conclusion holds in general for times of order up to, but not including $1 / \epsilon$ since the averages (16) may be achieved very slowly [30]. To make meaningful conclusions about the behavior of system (13) we need results that hold for times of order $1 / \epsilon$ and hence system (17) may not always be useful for studying the phase locking properties of the unaveraged system. We will provide examples of such cases in the next Section and first study the case when (17) is valid over long times.

Under the assumptions in (15) the averages (16) may be computed as

$$
\bar{\eta}=\sum_{i=1}^{m} \gamma_{i} \eta_{i} \quad \bar{\Gamma}(\rho)=\sum_{i=1}^{m} \gamma_{i} \Gamma_{i}(\rho)
$$

where $\gamma_{i}=\mu\left(P_{i}\right)$, and $m$ is the number of sets in the partition.

In order to understand how phase locking occurs when the averaged system (17) accurately describes the dynamics of the original system, we consider a few examples. We will assume that there are only two elements in the partition of $\Sigma$, as examples with more elements in the partition can be constructed similarly. We will use $x_{n+1}=F\left(x_{n}\right)=4 x_{n}\left(1-x_{n}\right)$, the logistic map on the interval $\Sigma=[0,1]$, and the partition $P_{1}=[0,1 / 2)$ and $P_{2}=[1 / 2,1]$, so that $\gamma_{1}=\gamma_{2}=1 / 2$ in (18). This is a noninvertible map, and thus the special flow defined in equation (8) is only a semi-flow. However the logistic map is similar to the Poincaré map obtained from the the Rössler system and the fact that it is noninvertible has no effect on the conclusions of this Section. For simplicity we also assume that $T=2 \pi$ so that $\rho \in[0,2 \pi)$.

We first consider an example for which $\eta_{1}=\eta_{2}=0$, corresponding to zero phase diffusion. Let

$$
\Gamma_{1}=\frac{1}{2} \cos (\rho)+1 \quad \Gamma_{2}=\frac{1}{2} \cos (\rho)-1
$$


As shown in Fig. 2(a), the average $\bar{\Gamma}(\rho)=\frac{1}{2} \Gamma_{1}(\rho)+\frac{1}{2} \Gamma_{2}(\rho)$ has a real root, and therefore the system will typically be phase locked with the drive.

If we choose $\Gamma_{1}=\cos (\rho)$ and $\Gamma_{2}=-.2+\cos (\rho+\pi)$ the average $\bar{\Gamma}$ does not have a real root, as illustrated in Fig. 2(b), and therefore the phase difference $\rho$ will on average decrease for typical orbits $\left\{x_{n}\right\}_{n=0}^{\infty}$.

In both of these examples the effective, long time response to a perturbation as given by the averaged equation, is very different from the instantaneous response. We note that in both cases our conclusions hold only for typical orbits of the Poincaré map $x_{n+1}=F\left(x_{n}\right)$, i.e. orbits that spend approximately equal times in $P_{1}$ and $P_{2}$, and for which the average $1 / N \sum_{n=0}^{\infty} \Gamma\left(\rho, x_{n}\right)$ is achieved quickly. Some atypical orbits, such as periodic orbits which spend long times in either $P_{1}$ or $P_{2}$, do not satisfy these conditions and may lead to very different behavior. This situation is reminiscent of periodically perturbed phase oscillators where certain unlikely realizations of the stochastic process may give atypical results [10].

As shown in Section 4, the difference in the return times to the section $\Sigma$ leads to the stochastic behavior of the phase and hence phase diffusion. The dependence of $\Gamma(\rho, x)$ on $x$ is due to the differences in the phase sensitivity along the section $\Sigma_{\phi_{0}}=\left\{\left(\phi_{0}, x\right) \mid \phi_{0}=\right.$ const. $\}$. To illustrate the effect of these terms, and the resulting similarities and differences of our model as compared with a noisy periodic oscillator in their response to a periodic perturbation, we consider three examples.

In all examples we assume that the periodic perturbation in (9) has the form $p\left(t, \phi, x_{n}\right)=c \tilde{p}\left(t, \phi, x_{n}\right)$, so that $c$ may be thought of as the strength of the driving signal. As a result we have $\Gamma(\rho, x)=c \tilde{\Gamma}(\rho, x)$, and we can examine how the model system responds to driving signals of varying strengths. For the following examples we will fix $\epsilon=.01$ and vary $c$ between 0.8 and 9.6.

In our first example we choose $\Gamma_{1}=\Gamma_{2}=c \cos (\rho)$, and let $\eta_{1}=.04$ and $\eta_{2}=$ -.04. When $c=0$ the behavior of the phase difference $\rho_{n}$ is similar to a random walk. For $c>0$, since

$$
\left|\rho_{n+1}-\frac{\pi}{2}\right|=\left|\eta\left(x_{n}\right)+\rho_{n}+\Gamma\left(\rho_{n}\right)-\frac{\pi}{2}\right|
$$

the region $I_{c}=\left\{\rho|| c \cos \rho \mid<\max \left(\eta_{1} / \epsilon, \eta_{2} / \epsilon\right)\right\}$ is an absorbing region for $\rho$. Phase locking is thus typically achieved after a certain number of transient oscillations $N_{T}$. Since the region $I_{c}$ shrinks with an increase in coupling strength $c$, the variance of the distribution of phase differences $\left\{\rho_{n}\right\}_{n=N_{T}}^{\infty}$ will also decrease, as illustrated in Fig. 3(a). As in the case of noisy periodic oscillators, an increase in coupling strength leads to tighter phase locking between the drive and response systems.

For our next example we choose $\eta_{1}=\eta_{2}=0$ and $\Gamma_{1}=c(1 / 2 \cos (\rho)+1)$ and $\Gamma_{2}=c(1 / 2 \cos (\rho)-1)$. Since $\eta(x)=0$ there is no phase diffusion in the unperturbed system. The average is $\bar{\Gamma}=c / 2 \cos (\rho)$, so that for any finite coupling strength 


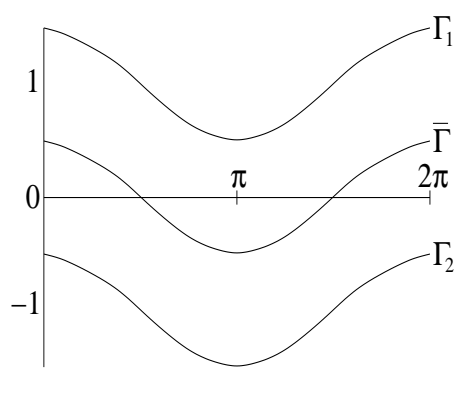

(a)

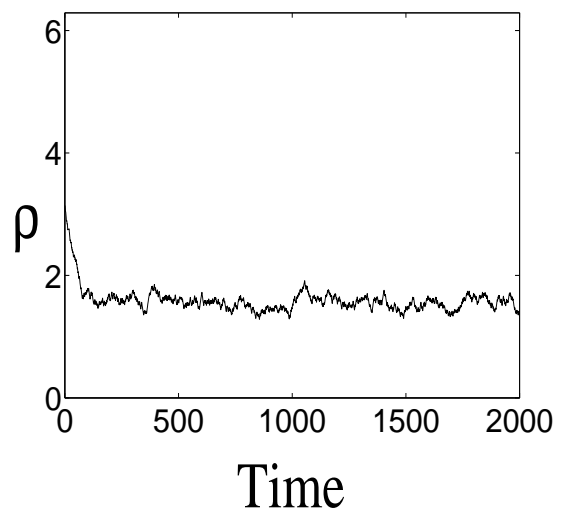

(c)

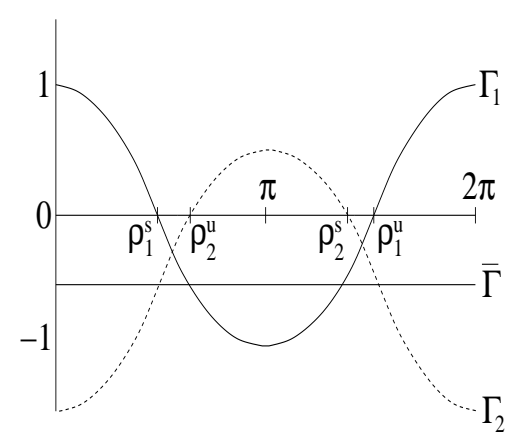

(b)

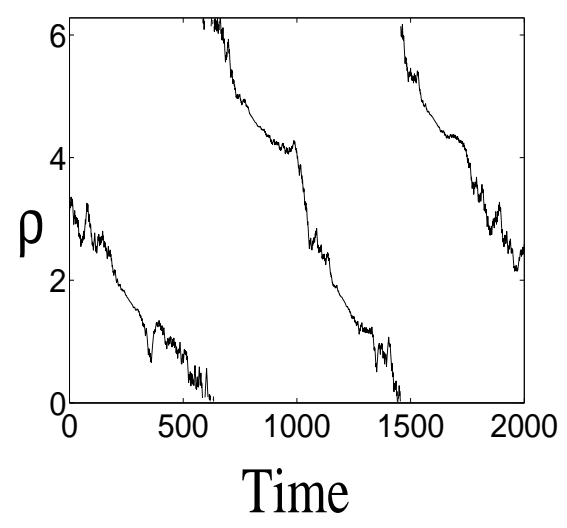

(d)

Figure 2: a) The graphs of $\Gamma_{1}=1 / 2 \cos (\rho)+1, \Gamma_{2}=1 / 2 \cos (\rho)-1$, and $\bar{\Gamma}$ for the first example. b) The graphs of $\Gamma_{1}=\cos (\rho), \Gamma_{2}=-.2+\cos (\rho+\pi)$, and $\bar{\Gamma}$ for the second example. c) The evolution of $\rho$ corresponding to the first example, when phase locking occurs. d) The evolution of $\rho$ corresponding to the second example, when phase locking does not occur. 


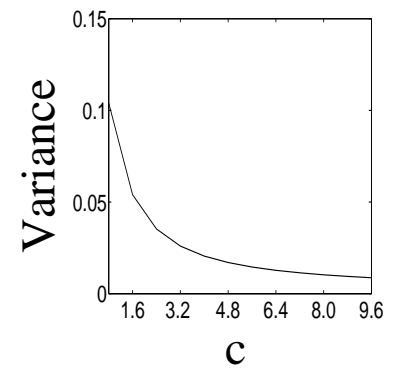

(a)

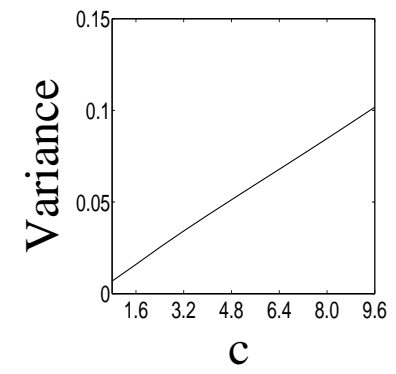

(b)

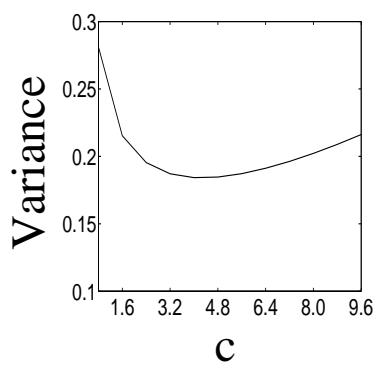

(c)

Figure 3: The variance of $\rho$ as a function of the coupling strength $c$. In a) $\Gamma_{1}=\Gamma_{2}=$ $c \cos (\rho), \eta_{1}=.04$ and $\eta_{2}=-.04$. In b) $\Gamma_{1}=c(1 / 2 \cos (\rho)+1), \Gamma_{2}=c(1 / 2 \cos (\rho)-1)$ and $\eta_{1}=\eta_{2}=0$. In c) $\Gamma_{1}=c(1 / 2 \cos (\rho)+1), \Gamma_{2}=c(1 / 2 \cos (\rho)-1), \eta_{1}=.04$ and $\eta_{2}=-.04$. The convergence to the trapping region is very slow for small values of the coupling parameter $c$, and they have thus been omitted from the figures.

typical orbits are attracted to $\pi / 2$. However, the step size $\left|\rho_{n+1}-\rho_{n}\right|$ increases with the coupling strength, and hence the variance of the distribution of $\left\{\rho_{n}\right\}_{n=N_{t}}^{\infty}$ will increase with the coupling strength, as illustrated in Fig. 3(b).

Therefore in these two examples the increase in coupling strength will have exactly the opposite effect. In the first case it leads to a tighter phase locked state, while in the second it leads to desynchronization.

In a typical system it is expected that both $\eta(x)$ and the phase responses $\Gamma(\rho, x)$ vary with $x$, and that the effect of an increase in coupling is a combination of these two. Our third example is of this type and is illustrated in Fig. 3(c). We take $\eta_{1}=.04, \eta_{2}=-.04$, and $\Gamma_{1}=c(1 / 2 \cos (\rho)+1), \Gamma_{2}=c(1 / 2 \cos (\rho)-1)$. Since the evolution of the phase difference is governed by $\rho_{n+1}=\rho_{n}+\eta\left(x_{n}\right)+c \Gamma\left(\rho, x_{n}\right)$, we may typically expect that for small coupling values, the effects of the term $\eta(x)$ will dominate, while for larger coupling values the effects of the term $\Gamma\left(\rho, x_{n}\right)$ play a more important role.

We emphasize that the shape of the graph in Fig. 3(c), indicates that typical systems of this type are expected to have the remarkable property that the most precise phase locked state occurs at a critical coupling strength. If the coupling strength is too low, the effects of the phase diffusion will tend to make synchrony imprecise. Similarly, if the coupling is too strong, the effects of the term $\Gamma(\rho, x)$ are going to affect the precision of synchrony. This effect has significant implications since the precision of synchrony is of fundamental importance in a number of areas. 


\section{Insufficiently fast convergence to the average}

As we have mentioned above, if the averages in (16) are not achieved sufficiently fast, then equation (17) may not provide a good approximation to the behavior of the original system for times of $\mathcal{O}(1 / \epsilon)$. In this Section we describe a class of systems which illustrate why the approach discussed in the previous Section may not be valid in general. In particular, examples can be constructed of systems for which an increase in the amplitude of the periodic perturbation may lead to desynchronization in such a way that the frequency of phase slips increases with the size of the perturbation [31]. This type of system therefore also responds very differently to periodic forcing than chaotic systems that can be treated as noisy limit cycles $[11,4]$.

Consider a system where there are long subsequences in typical orbits of the Poincaré map that remain mostly in one of the partition elements. Also suppose that the the fixed points of the functions $\Gamma_{i}, i=1,2$, are oriented so that the stable fixed points are separated by the unstable ones. While the orbit of the Poincaré map is in the first element of the partition, the phase difference will approach the stable fixed point of $\Gamma_{1}$. When the orbit moves into the second partition element, the phase difference will approach the stable fixed point of $\Gamma_{2}$, but because of the positioning of the unstable fixed points, it must move around the entire circle in doing so. Each time the orbit moves into a new element of the partition, the phase difference will move around the circle in this manner. We will refer to this process as slipping. More formally

Definition 7.1 The phase difference $\Psi(t)$ between two oscillators is slipping if the lift of $\Psi(t)$ to the line can be bounded above (below) by a function $F: \mathbb{R} \rightarrow \mathbb{R}$, such that $F^{\prime}<-K<0\left(F^{\prime}>K>0\right)$, for some constant $K>0$.

We now make these ideas more precise. Let us again assume that $\rho$ evolves according to

$$
\begin{aligned}
& \rho_{n+1}=\rho_{n}+\eta\left(x_{n}\right)+\epsilon \Gamma\left(\rho_{n}, x_{n}\right)=\rho_{n}+\epsilon H\left(\rho_{n}, x_{n}\right) \\
& x_{n+1}=F\left(x_{n}\right)
\end{aligned}
$$

We again assume that $H\left(\rho, x_{n}\right)$ achieves finitely many values for a fixed $\rho$ so that $H\left(\rho, x_{n}\right)=H_{i}(\rho)$ if $x_{n} \in P_{i}$ and that the functions $\rho+\epsilon H_{i}(\rho)$ are invertible. For a fixed $i$, let $\left\{\rho_{i, k}^{s}\right\}_{k=1}^{r_{i}}$ and $\left\{\rho_{i, k}^{u}\right\}_{k=1}^{r_{i}}$ be the stable and unstable fixed points respectively of the system $\rho_{n+1}=\rho_{n}+\epsilon H_{i}\left(\rho_{n}\right)$, i.e. the zeroes of $H_{i}(\rho)$ with $H_{i}^{\prime}\left(\rho_{i, k}^{s}\right)<0$ and $H_{i}^{\prime}\left(\rho_{i, k}^{u}\right)>0$. For simplicity we also assume that $\rho_{i, t}^{s} \neq \rho_{j, v}^{u}$ for any $i \neq j$ or $t \neq v$, and that the zeroes are a distance $\mathcal{O}(1)$ apart (this follows from the assumption that the $H(\rho)$ are independent of $\epsilon)$. Given an interval $I=[a, b]$ we say that the orbit of 


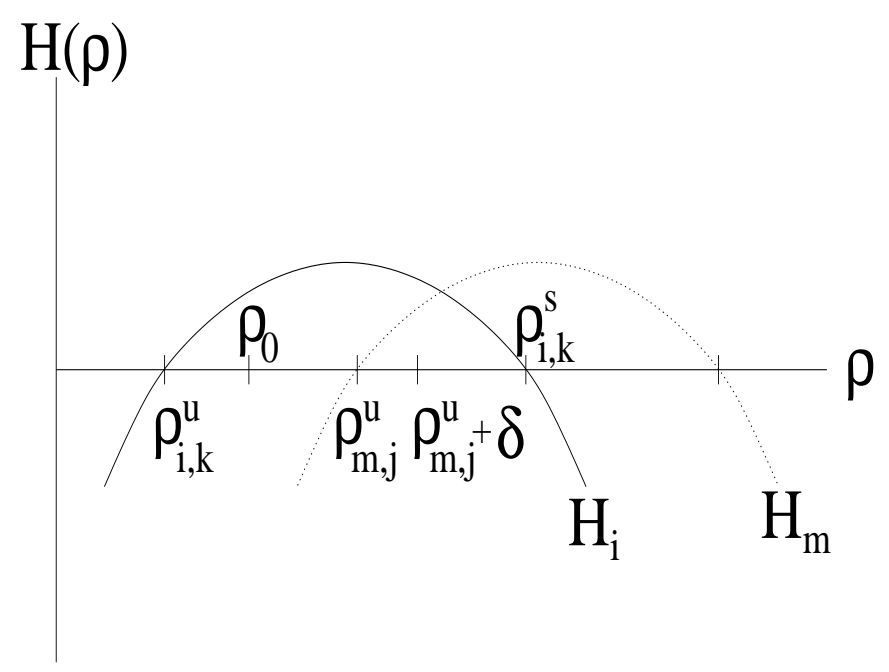

Figure 4: An illustration of the proof of proposition 7.2. For a sequence $y_{0}, \ldots, y_{N_{1}}, y_{N_{1}+1}, \ldots, y_{N}$ the orbit of $\rho_{0}$ will first move past $\rho_{m, j}^{u}+\delta$ at the $N_{1}$-step, and past $\rho_{i, k}^{S}$ at the $N$-th step.

a point $\rho_{0} \in I$ leaves $I$ through the endpoint $b$ under the dynamics of (20) if there exists an $N$ such that $\rho_{0}, \ldots, \rho_{N} \in I$ and $\rho_{N+1}, \ldots, \rho_{M}>b$ for some $M>N$.

Proposition 7.2 Let $I_{k}=\left[\rho_{i, k}^{u}, \rho_{i, k}^{s}\right]$. If there exists an $m$ such that $H_{m}\left(\rho_{i, k}^{s}\right)>0$, then for any $\rho_{0} \in I_{k}$ there exists a sequence $y_{0}, \ldots, y_{N}$ such that $\rho_{n}$ leaves $I_{k}$ through $\rho_{i, k}^{s}$ under the dynamics of

$$
\rho_{n+1}=\rho_{n}+\epsilon H\left(\rho_{n}, y_{n}\right)
$$

The idea of the proof is illustrated in Fig. 4. The orbit of the point $\rho_{0}$ will move past $\rho_{m, j}^{u}+\delta$ given a sufficiently long sequence $y_{0}, \ldots, y_{N_{1}} \in P_{i}$. Once in the interval $\left[\rho_{m, j}^{u}+\delta, \rho_{i, k}^{s}\right]$, the orbit of $\rho_{0}$ will move past $\rho_{i, k}^{s}$ given a sufficiently long sequence $y_{N_{1}}, \ldots, y_{N_{2}} \in P_{m}$. A full proof may be found in the Appendix. Since the zeroes the $H_{i}$ are $\mathcal{O}(1)$ apart in $\epsilon$ a similar argument may be used to show that the orbit of any $\rho_{0} \in I_{k}$ will leave an interval $\left[\rho_{i, k}^{u}, \rho_{i, k}^{s}+\delta_{2}\right]$ through $\rho_{i, k}^{s}+\delta_{2}$ for some $\delta_{2}=\mathcal{O}(1)$ in $\epsilon$. It follows that the conclusion of Proposition 7.2 also holds for the unaveraged system (11) for sufficiently small $\epsilon$. A similar argument also gives a lower bound on the number of iterates necessary to leave $I$ which is also of order $1 / \epsilon$.

We note that the sequence $y_{0}, \ldots, y_{N}$ constructed in the proof needs to actually occur in the orbit of $x_{0}$ under $x_{n+1}=F\left(x_{n}\right)$ for the conclusions of Proposition 7.2 
to hold in an actual example. Since the number of steps necessary to leave the interval $I$ is of order $1 / \epsilon$ it diverges as $\epsilon \rightarrow 0$. Therefore the necessary sequences become longer, and are less likely to occur. However, if, for instance, the sets $P_{i}$ give a Markov partition of $\Sigma$ that leads to a full shift on $n$ symbols, or even a subshift which contains sequences of the type described in Proposition 7.2, then the probability that such sequences do occur may be finite for any $\epsilon>0$.

The following Theorem shows that if such special sequences occur frequently, then slipping may occur even if the corresponding averaged system (17) discussed in the previous Section has stable fixed points.

Theorem 7.3 The orbit of $\rho_{0} \in[0, T)$ can slip in an increasing (decreasing) direction if and only if for every $\rho_{i, j}^{s} \in[0, T)$ there exists an $m$ such that $H_{m}\left(\rho_{i, j}^{s}\right)>0$ $\left(H_{j}\left(\rho_{i, j}^{s}\right)<0\right)$.

The proof of the Theorem consists in noting that the conditions imply that an orbit can move past the barriers posed by the points $\rho_{i, j}^{s}$. As a direct corollary to the theorem we have sufficient conditions under which slipping is not possible

Corollary 7.4 If there exist points $\rho_{i, j}^{s}$ and $\rho_{k, l}^{s}$ such that $H_{m}\left(\rho_{i, j}^{s}\right) \leq 0$ and $H_{m}\left(\rho_{k, l}^{s}\right) \geq$ 0 for all $m$ then slipping is not possible.

This corollary is not surprising as the points $\rho_{i, j}^{s}$ and $\rho_{k, l}^{s}$ form barriers to the orbit of $\rho$. This conclusion holds in much more general systems. It is easy to check that if there exist regions $I_{1}, I_{2} \in S^{1}$ of size $\mathcal{O}(1)$ such that $\eta\left(x_{n}\right)+\Gamma\left(\rho, x_{n}\right) \geq 0$ for all $\rho \in I_{1}$ and all $x_{n}$, and $\Delta\left(x_{n}\right)+\Gamma\left(\rho, x_{n}\right) \leq 0$ for all $\rho \in I_{2}$ and all $x_{n}$, then the orbit of $\rho$ will be trapped between $I_{1}$ an $I_{2}$ under the dynamics of (13).

One of the surprising consequences implicit in these results is that the length of the sequences $y_{0}, \ldots, y_{n}$ necessary to induce phase slipping was dependent on $\epsilon$. In particular, the larger the coupling strength, the shorter the length of the sequence necessary to induce phase slipping, and the quicker the phase slips. This leads to the somewhat surprising result that an increase in coupling strength is expected to lead to an increase in the frequency and speed of phase slips in the types of systems described in this Section. It is straightforward to construct model system demonstrating this behavior using the example described in [31]. Using the ideas of Theorem 7.3 it is possible to construct examples in which phase slips occur in both directions.

The situation described in this section may seem unrealistic from the physical viewpoint. It appears necessary to assume that the time the system spends in each element of the partition and the rate of approach to the fixed points of the individual functions $\Gamma_{i}$ need to be related. Since the rate of convergence to the fixed points is proportional to the forcing strength this would imply a relation between the coupling 
strength and the dynamics of the return map, which does not seem typical. There are at least two physically realistic situation in which such an assumption is not necessary:

Suppose we have a system in which the switching time between the elements in the partition is slow relative to the oscillation frequency. For very small coupling values phase diffusion can be expected to dominate. For somewhat larger coupling values, averaging may be appropriate since the time necessary to arrive to a fixed point of one of the functions $\Gamma_{i}$ may be long compared to the switching time between the partitions. However, as the coupling is increased, these equilibria are approached at a faster rate, and we enter the regime described in this chapter. Of course, it is necessary to assume that the forcing is still sufficiently small so that the arguments we have presented hold.

Another physical situation in which these observations may be of interest is when the distribution of switching times between the elements of the partition has a heavy tail. In this case arbitrarily long switching times may be observed, and the situation described in this chapter may be relevant even for relatively small couplings.

\section{Conclusions}

We have shown how the theory of phase locking can be extended to systems with phase coherent chaotic attractors. Under the simplifying assumption that the system is subject to a periodic perturbation affecting only its phase variable, equations for the phase difference can be reduced to a skew-product of a circle map with a chaotic diffeomorphism. This is a natural generalization of the case of coupled periodic oscillators in which the dynamics of the phase difference can be described by a circle map.

The assumption that the periodic perturbation affects only the phase was motivated by the fact that the phase direction is nearly neutral due to the assumption of phase coherence. Hence the effect of a perturbation will be greatest in this direction. We therefore believe that the ideas we present hold for more general types of coupling that affect the dynamics of the radial variable, although to analyze this case the second equation in (23) would have to be replaced by $x_{n+1}=F\left(x_{n}, \rho_{n}\right)$. Such systems are considered in [32]. We also note that using the ideas presented in [17], many of the ideas presented here can be extended to bidirectionally coupled chaotic oscillators.

We note that in our definition of phase locking we require that $\left|\rho_{n}-\theta\right|<C<$ $T$. The constant $C$ therefore gives an upper bound on the deviation of the phase difference from $\theta$. In some applications it may be necessary to require precise phase locked states, and thus small values of $C$. In such cases, the phase difference $\rho_{n}$ 
could be trapped in a subset of $[0, T)$, and yet for practical purposes the system would not be phase locked. The examples of Sections 6 and 7 show that for certain chaotic systems we may expect that optimal coupling values lead to the tightest synchronization.

We have studied model systems in which the effects of the different terms in equation (13) can be isolated and analyzed independently. In typical chaotic phase coherent systems we expect that all the effects we have described separately play a role in the evolution of the phase difference. Thus the study of phase locking in realistic systems will be more complex, and depend on the structure of the chaotic attractor and the nature on the perturbation. We have indicated that such systems may exhibit very rich behavior, which may be quite different from that observed in periodically perturbed stochastic oscillators. It is possible that phase coherent biological oscillators may benefit from having access to such a richness of responses to different stimuli.

Theorem 5.1 and the discussion in Section 6 suggest that a similar approach may be adopted in computing phase response curves for impulsively perturbed chaotic oscillators [14]. In [33] it was observed that a chaotic oscillator may be phase locked with a periodic sequence of pulses, even when the corresponding phase response curve indicates there are no strict trapping regions for the phase difference. We are currently examining whether this observation can be explained by considering an appropriate average of phase response curves.

We thank the Max Planck Institute for Physics of Complex Systems for their generous hospitality in hosting the workshop "Control, Communication, and Synchronization in Chaotic Dynamical Systems" during which this work was first presented. We also thank G. R. Hall and J. Ritt for stimulating discussions.

\section{Appendix}

Proof of Proposition 3.2: Let $y(t, x)$ be the solution of $y^{\prime}=f(y)$ such that $y(0, x)=x \in \Sigma$. Let $R: N \rightarrow \mathbb{R}^{n-1}$ be any function such that $R(y(0, x))=$ $x, R(y(T(x), x))=F(x)$ and $R(y(T(x)+t, x))=R(y(t, F(x)))$ where $R(y(t, x))$ depends smoothly on both $t$ and $x$. $R$ may be chosen to have these properties due to smooth dependence on initial conditions.

Similarly we choose a function $\tilde{\phi}: N \rightarrow \mathbb{R}$ such that $\tilde{\phi}(y(0, x))=0, \tilde{\phi}(y(T(x), x)=$ $T$ and $\tilde{\phi}(y(T(x)+t, x)=\tilde{\phi}(y(t, F(x))+T$ so that $\tilde{\phi}(y(t, x))$ again depends smoothly on both $t$ and $x$. Note that we can choose $\tilde{\phi}$ such that $\frac{\partial \tilde{\phi}}{\partial t}$ is close in the $L^{1}$ norm to $\frac{T}{T(x)}$ for $t \in[0, T(x)]$.

By construction $\tilde{\phi}$ is the lift of a phase variable $\phi \in S^{1}$ to the line, so that $\phi$ and $R$ define a new coordinate system in the vicinity of the attractor and the equations 
of motion in these new coordinates satisfy (4-5).

Proof of Theorem 5.1: Without loss of generality we will assume that the initial conditions for system (9), and hence system (11) lie on $\Sigma$, since we can always flow forward to the next intersection. The proof proceeds in two steps. In the first step we will use estimates of an appropriate average of system (11) over a single excursion from $\Sigma$ back to $\Sigma$ to reduce the system. The second part consists of the discretization of the reduced system.

By definition, the orbit of the full system lies on $\Sigma$ when $\phi=0$. By equation (10) this means that at the time of crossing $\Psi=-\frac{T}{T^{\prime}} t \bmod T$, or $t=-\frac{T^{\prime}}{T} \Psi+N T^{\prime}$, for some N. Since the function $p$ is $T^{\prime}$ periodic in the first variable we can rewrite equation (11) for $S_{n-1}^{0} \leq \tilde{\phi}<S_{n}^{0}$ as

$$
\Psi^{\prime}=\Delta\left(x_{n}\right)+\epsilon p\left(-\frac{T^{\prime}}{T} \Psi+t, \frac{T\left(x_{n}\right)}{T^{\prime}} t, x_{n}\right)+\mathcal{O}\left(\epsilon^{2}\right)
$$

To simplify notation we will therefore define a new function

$$
G\left(t, \Psi, x_{n}\right)=p\left(-\frac{T^{\prime}}{T} \Psi+t, \frac{T\left(x_{n}\right)}{T^{\prime}} t, x_{n}\right) .
$$

Our first step will be to show that the original system can be reduced to

$$
\begin{aligned}
\bar{\rho}^{\prime} & =\Delta\left(x_{n}\right)+\epsilon \bar{G}\left(\bar{\rho}, x_{n}\right) & & \text { for } S_{n-1}^{0} \leq \tilde{\phi}<S_{n}^{0} \\
x(t) & =x_{n}=F\left(x_{n-1}\right) & & \text { for } S_{n-1}^{0} \leq \tilde{\phi}<S_{n}^{0} .
\end{aligned}
$$

using averaging.

Following the proof of the Averaging Theorem in [34] we first define a new variable $\bar{\Psi}$ on each segment $\tilde{\phi} \in\left[S_{n}^{0}, S_{n+1}^{0}\right)$ by

$$
\Psi=\bar{\Psi}+\epsilon w\left(t, \bar{\Psi}, x_{n}\right)
$$

where

$$
w\left(t, \bar{\Psi}, x_{n}\right)=\int_{0}^{t} \tilde{G}\left(s, \bar{\Psi}, x_{n}\right) d s
$$

and $\tilde{G}$ is the oscillatory part of $G$, i.e. $\tilde{G}(t, \Psi, x)=G(t, \Psi, x)-\bar{G}(\Psi, x)$ and $\bar{G}\left(\Psi, x_{n}\right)=\left(1 / T^{\prime}\right) \int_{0}^{T^{\prime}} G\left(t, \Psi, x_{n}\right) d t=\left(1 / T^{\prime}\right) \Gamma\left(\Psi, x_{n}\right)$ is the average of $G$. As in the proof of the Averaging Theorem, $\frac{\partial w}{\partial t}=\tilde{G}\left(t, \Psi, x_{n}\right)$, where we have chosen the constant of integration to be 0 . Following the estimates in [34] we can conclude that

$$
\bar{\Psi}^{\prime}=\epsilon \bar{G}\left(\bar{\Psi}, x_{n}\right)+\epsilon^{2} H\left(t, \bar{\Psi}, x_{n}\right)+\Delta\left(x_{n}\right)
$$


where $H=\mathcal{O}(1)$. The solutions of $(26)$ remain $\mathcal{O}(\epsilon)$ close to solutions of the averaged equation

$$
\bar{\rho}^{\prime}=\epsilon \bar{G}\left(\bar{\rho}, x_{n}\right)+\Delta\left(x_{n}\right)
$$

for all times $\tau$ such that $\left|T^{\prime}-\tau\right|=\mathcal{O}(\epsilon)$ if $|\bar{\rho}(0)-\bar{\Psi}(0)|=\mathcal{O}(\epsilon)$.

Let $T_{n}^{\epsilon}=T_{n}^{\epsilon}(x(0), 0)$ be the $n$-th return time to the section $\Sigma$ of the perturbed system (note that we start our count with 0 , so that the first excursion ends at the 0 -th return time $\left.T_{0}^{\epsilon}\right) .\left|T_{n}^{\epsilon}-T\right|=\mathcal{O}(\epsilon)$ since the perturbation of the phase in (9) is $\mathcal{O}(\epsilon)$. Let $\Delta \bar{\Psi}_{n}=\bar{\Psi}\left(T_{n}^{\epsilon}\right)-\bar{\Psi}(0)$, and $\Delta \bar{\rho}_{n}=\bar{\rho}\left(T_{n}^{\epsilon}\right)-\bar{\rho}(0)$. If $|\bar{\Psi}(0)-\bar{\rho}(0)|=O(\epsilon)$ then we can estimate

$$
\left|\Delta \bar{\Psi}_{n}-\Delta \bar{\rho}_{n}\right| \leq \epsilon \int_{0}^{T_{n}^{\epsilon}}\left|\bar{G}\left(\bar{\Psi}(t), x_{n}\right)-\bar{G}\left(\bar{\rho}(t), x_{n}\right)\right| d t+\epsilon^{2} \int_{0}^{T_{n}^{\epsilon}}\left|H\left(t, \bar{\Psi}(t), x_{n}\right)\right| d t
$$

so that $\left|\Delta \bar{\Psi}_{n}-\Delta \bar{\rho}_{n}\right|=\mathcal{O}\left(\epsilon^{2}\right)$. This approximation shows that even if we make an $\mathcal{O}(\epsilon)$ error in choosing an initial condition, the error in estimating the $n$-th change in $\Psi$ is still only of order $\epsilon^{2}$.

Let $T_{0}^{\epsilon}+\ldots+T_{n}^{\epsilon}=S_{n}^{\epsilon}$, and define the sequence $\left\{\rho_{n}\right\}_{n=0}^{\infty}$ of approximations to the values $\Psi\left(T_{0}^{\epsilon}+\ldots+T_{n}^{\epsilon}\right)=\Psi\left(S_{n}^{\epsilon}\right)$ inductively by

$$
\rho_{n}=\bar{\rho}_{n-1}\left(T_{n-1}^{\epsilon}\right)
$$

where $\bar{\rho}_{n-1}$ are solutions of $(27)$ with initial condition $\bar{\rho}_{n-1}(0)=\rho_{n-1}$, and $\rho_{0}=$ $\Psi(0)$. Note that since $\left|T_{n}^{\epsilon}-T^{\prime}\right|=\mathcal{O}(\epsilon)$

$$
\begin{aligned}
w\left(T_{n}^{\epsilon}, \bar{\Psi}, x_{n}\right) & =\int_{0}^{T_{n}^{\epsilon}} G\left(t, \bar{\Psi}, x_{n}\right) d t-\frac{T_{n}^{\epsilon}}{T^{\prime}} \int_{0}^{T^{\prime}} G\left(t, \bar{\Psi}, x_{n}\right) d t \\
& =\int_{T^{\prime}}^{T_{n}^{\epsilon}} G\left(t, \bar{\Psi}, x_{n}\right) d t+\mathcal{O}(\epsilon)=\mathcal{O}(\epsilon) .
\end{aligned}
$$

Let $\Psi_{n}(t)$ be the solution of equation (11) between the $n$-th and $n+1$-st return to the section $\Sigma$, so that $\Psi_{n}(t)=\Psi\left(S_{n}^{\epsilon}+t\right)$ and let $\Delta \Psi_{n}=\Psi_{n}\left(T_{n}^{\epsilon}\right)-\Psi_{n}(0)$. Similarly we define $\Delta \rho_{n}=\rho_{n+1}-\rho_{n}$. If $\left|\rho_{n}-\Psi_{n}(0)\right|=\mathcal{O}(\epsilon)$ then

$$
\begin{aligned}
\left|\Delta \Psi_{n}-\Delta \rho_{n}\right| & =\left|\bar{\Psi}\left(T_{n}^{\epsilon}\right)-\bar{\Psi}(0)-\bar{\rho}_{n}\left(T_{n}^{\epsilon}\right)+\bar{\rho}_{n}(0)+\epsilon w\left(T_{n}^{\epsilon}, \bar{\Psi}\left(T_{n}^{\epsilon}\right), x_{n}\right)\right| \\
& =\left|\Delta \bar{\Psi}_{n}-\Delta \bar{\rho}_{n}\right|+\mathcal{O}\left(\epsilon^{2}\right)=\mathcal{O}\left(\epsilon^{2}\right)
\end{aligned}
$$

where $\bar{\Psi}$ is the solution of $(26)$ with $\bar{\Psi}(0)=\Psi_{n}(0)$. Using induction and a repeated application of the triangle inequality it follows that

$$
\left|\Psi_{n}(0)-\rho_{n}-\Psi(0)+\rho_{0}\right|=\left|\Psi\left(S_{n}^{\epsilon}\right)-\rho_{n}\right|=\mathcal{O}(\epsilon)
$$


for $n=\mathcal{O}(1 / \epsilon)$. By the estimates above it also follows that $\rho$ and $\Psi$ are $\mathcal{O}(\epsilon)$ close for times of order 1 if $\left|\rho\left(S_{n}^{\epsilon}\right)-\Psi\left(S_{n}^{\epsilon}\right)\right|=\mathcal{O}(\epsilon)$, and we can therefore conclude that the orbits of system (11) are $\mathcal{O}(\epsilon)$ close to the orbits of the system (23) for times of order $1 / \epsilon$.

We next reduce (23) to the discrete dynamical system (13). Starting our integration at the $n$-th return to the section $\Sigma$ and ending at time $T_{n}^{\epsilon}$ afterwards an application of Gronwall's Inequality yields

$$
\begin{aligned}
\rho_{n+1} & =\rho_{n}+\Delta\left(x_{n}\right) T_{n}^{\epsilon}+\epsilon T_{n}^{\epsilon} \bar{G}\left(\rho_{n}, x_{n}\right)+O\left(\epsilon^{2}\right) \\
& =\rho_{n}+\Delta\left(x_{n}\right) T^{\prime}+\epsilon T^{\prime} \bar{G}\left(\rho_{n}, x_{n}\right)+\mathcal{O}\left(\epsilon^{2}\right)
\end{aligned}
$$

where we have again used the fact that $\left|T_{n}^{\epsilon}-T^{\prime}\right|=O(\epsilon)$. The conclusion of the theorem follows when we let $\eta\left(x_{n}\right)=\Delta\left(x_{n}\right) T^{\prime}$, define

$$
\Gamma\left(\rho_{n}, x_{n}\right)=T^{\prime} \bar{G}\left(\rho_{n}, x_{n}\right),
$$

and, by a slight abuse of notation, drop the $\mathcal{O}\left(\epsilon^{2}\right)$ terms in equation (31).

Proof of Proposition 5.2: Assume that $I_{2} \subset I_{1}$ are absorbing regions with the desired properties for the system

$$
\begin{aligned}
\rho_{n+1} & =\zeta\left(x_{n}\right)+\Gamma\left(\rho_{n}, x_{n}\right) \\
x_{n+1} & =F\left(x_{n}\right)
\end{aligned}
$$

for any $\rho_{0} \in S^{1}$ and a particular $x_{0} \in \Sigma$. Then there exists a time $\tau$ after which any orbit in $S^{1}$ is trapped in $I_{2}$. The same conclusion holds for system

$$
\begin{aligned}
\rho_{n+1} & =\epsilon \zeta\left(x_{n}\right)+\epsilon \Gamma\left(\rho_{n}, x_{n}\right) \\
x_{n+1} & =F\left(x_{n}\right)
\end{aligned}
$$

with trapping time $\tau / \epsilon$. By Theorem 5.1, if (32) is obtained from (11) then for sufficiently small $\epsilon$ we have $\left|\Psi_{n}-\rho_{n}\right|<\delta(\epsilon)=\mathcal{O}(\epsilon)$ for the time $\lceil\tau / \epsilon\rceil=\tilde{\tau}$. Here $\lceil r\rceil$ denotes the smallest integer greater than $r$, and $\Psi_{n}=\Psi\left(S_{n}^{\epsilon}\right)$ where $S_{n}^{\epsilon}$ is the time of the $n$-th return to $\Sigma$, as in Theorem 5.1.

Let $\rho_{n}\left(\rho_{0}\right)$ denote the $\rho$ coordinate of the $n$-th iterate in the orbit of (32) starting at $\left(\rho_{0}, x_{0}\right)$, and let $\nu_{0}=\Psi(0)$ and

$$
\nu_{n}=\rho_{\tilde{\tau}}\left(\Psi_{(n-1) \tilde{\tau}}\right) .
$$

Therefore $\left|\nu_{n}-\Psi_{n \tilde{\tau}}\right|<\delta(\epsilon)$. Since any orbit starting in $S^{1}$ reaches $I_{2}$ within the time $\tilde{\tau}$ it follows that $\nu_{n} \in I_{2}$ so that $d\left(I_{2}, \Psi_{n \tilde{\tau}}\right)<\delta(\epsilon)$ for all $n$. Hence for sufficiently small $\epsilon$ the iterates $\Psi_{n \tilde{\tau}}$ are in $I_{1}$. Since $I_{1}$ is an absorbing region for (32) it follows 
that $\rho_{n}\left(\Psi_{n \tilde{\tau}}\right)$ is contained in $I_{1}$ for all $0<n<\tilde{\tau}$, and hence $d\left(\Psi_{n}, I_{1}\right)<\delta(\epsilon)$ for all $n$.

Proof of Proposition 7.2: If $H_{m}(\rho)>0$ for all $\rho \in\left[\rho_{0}, \rho_{i, k}^{s}\right]$, let $M_{m}=$ $\min _{\rho \in\left[\rho_{0}, \rho_{i, k}^{s}\right]} H_{m}(\rho)$. Let $y_{0}, \ldots y_{N}$ be a sequence such that $y_{i} \in P_{m}$ and $N=\left\lceil\frac{\left|I_{k}\right|}{\epsilon M_{m}}\right\rceil$ where $\left|I_{k}\right|$ is the length of the interval $I_{k}$. Then the orbit of any $\rho_{0} \in I_{k}$ leaves $I_{k}$ through $\rho_{i, k}^{s}$ in at most $N=\mathcal{O}(1 / \epsilon)$ steps.

If $H_{m}(\rho)=0$ at some points in $\left[\rho_{0}, \rho_{i, k}^{s}\right]$ let $\rho_{m, j}^{u} \in I_{k}$ be the rightmost such point. Choose a $\delta=\mathcal{O}(1)$ in $\epsilon$ such that $\rho_{m, j}^{u}+\delta \in\left[\rho_{m, j}^{u}, \rho_{i, k}^{s}\right]$. Let

$$
M_{i}=\min _{\rho \in\left[\rho_{0}, \rho_{m, j}^{u}+\delta\right]} H_{i}(\rho)
$$

and let $M_{m}=\min _{\rho \in\left[\rho_{m, j}^{u}+\delta, \rho_{i, k}^{s}\right]} H_{m}(\rho)$ so that $M_{i}, M_{m}>0$. Let $N_{1}=\left\lceil\mid\left[\rho_{0}, \rho_{m, j}^{u}+\right.\right.$ $\left.\delta] \mid /\left(\epsilon M_{i}\right)\right\rceil$, and $N_{2}=\left\lceil\left|\left[\rho_{m, j}^{u}+\delta, \rho_{i, k}^{s}\right]\right| /\left(\epsilon M_{m}\right)\right\rceil$. Choose a sequence so that $y_{0}, \ldots, y_{N_{1}} \in$ $P_{i}$ and $y_{N_{1}+1}, \ldots, y_{N_{1}+N_{2}} \in P_{m}$. Then the orbit of $\rho_{0}$ leaves $I_{k}$ in at most $N=$ $N_{1}+N_{2}=\mathcal{O}(1 / \epsilon)$ steps.

Proof of Theorem 7.3: If there exists an $m$ such that $H_{m}\left(\rho_{i, j}^{s}\right)>0$ for every $\rho_{i, j}^{s}$, then the circle $S^{1}=[0, T) / \sim$ can be covered by finitely many intervals $\left\{I_{n}\right\}_{n=1}^{K}$ where $I_{n}=\left[\rho_{i, k}^{u}, \rho_{i, k}^{s}\right]$ for some $i$ and $k$ such that the conditions of Proposition 7.2 are satisfied on each $I_{n}$. We obtain the desired result by concatenating the sequences $y_{N_{i}}, \ldots, y_{N_{i+1}}$ obtained in the proof of Proposition 7.2 to obtain a sequence $y_{0}, \ldots, y_{N_{1}+\ldots+N_{K}}$ such that the orbit $\rho_{0}$ will complete a full circle under the dynamics of $(22)$.

On the other hand if there exists a point $\rho_{i, j}^{s}$ such that $H_{m}\left(\rho_{i, j}^{s}\right) \leq 0$ for all $m$, then the orbit of any $\rho_{0}$ in $I=\left[\rho_{i, j}^{u}, \rho_{i, j}^{s}\right]$ cannot leave $I$ through $\rho_{i, j}^{s}$. Since the fixed points of the system are assumed to be $\mathcal{O}(1)$ apart in $\epsilon$ and the iterates $\rho_{n}$ are $\mathcal{O}(\epsilon)$ apart, this concludes the proof.

\section{References}

[1] C. Huygens. Horologivm oscillatorivm. Paris, 1673.

[2] A. Pikovsky. Phase synchronization of chaotic oscillations by a periodic external fields. Soviet J. Comm. Tech. Electronics, 30:85-90, 1985.

[3] E. Stone. Frequency entrainment of a phase coherent attractor. Phys. Lett. A, 163:47-50, 1992. 
[4] A. Pikovsky, M. Rosenblum, G. Osipov, and J. Kurths. Phase synchronization of chaotic oscillators by external driving. Physica D, 104:219-238, 1997.

[5] R. C. Elson, A. I. Selverston, R. Huerta, N. F. Rulkov, M. I. Rabinovich, and H. D. I. Abarbanel. Synchronous behavior of two coupled biological neurons. Phys. Rev. Lett., 81(25):5692-5695, 1998.

[6] V. Makarenko and R. Llinás. Experimentally determined chaotic phase synchronization in a neuronal system. Proc. Natl. Acad. Sci. USA, 95:15474-15752, 1998 .

[7] S. J. Schiff, K. Jerger, D.H. Duong, T. Chang, M L. Spano, and W. L. Ditto. Controlling chaos in the brain. Nature, 370:615-620, 1994.

[8] M. de Sousa Vieira. private communication.

[9] E. Rosa, W. Pardo, C. M. Ticos, J. A. Walkenstein, and M. Monti. Phase synchronization of chaos in a plasma discharge tube. Int. J. of Bifurcations and Chaos, 10:2551-2563, 2000.

[10] R. L. Stratonovich. Topics in the theory of random noise. Vol. II. Gordon and Breach Science Publishers, New York, 1963.

[11] A. Pikovsky, M. Zaks, M. Rosenblum, G. Osipov, and J. Kurths. Phase synchronization of chaotic oscillations in terms of periodic orbits. Chaos, 7(4):680-687, 1997.

[12] B.R. Hunt, E. Ott, and Jr. E. Rosa. Sporadically fractal basin boundaries of chaotic systems. Phys. Rev. Lett., 82(18):3597-3600, 1999.

[13] E. Rosa, E. Ott, and M. H. Hess. Transition to phase synchronization of chaos. Phys. Rev. Lett, 80(8):1642-1645, 1998.

[14] A. T. Winfree. The geometry of biological time. Springer-Verlag, Berlin, 1980.

[15] G.B. Ermentrout and N. Kopell. Oscillator death in systems of coupled neural oscillators. SIAM Journal of Applied Mathematics, 50:125-146, 1990.

[16] L. Glass and M. C. Mackey. From clocks to chaos. Princeton University Press, Princeton, NJ, 1988. The rhythms of life.

[17] Y. Kuramoto. Chemical oscillations, waves, and turbulence. Springer-Verlag, Berlin, 1984. 
[18] D. Farmer. Spectral broadening of period-doubling bifurcation sequences. Phys. Rev. Lett., 47(3):179-182, 1981.

[19] K. Josić and D.J. Mar. Phase synchronization of chaotic systems with small phase diffusion. Phys. Rev. E, 64:056234-1-056234-10, 2001.

[20] I. P. Cornfeld, S. V. Fomin, and Ya. G. Sină̌. Ergodic theory. Springer-Verlag, New York, 1982. Translated from the Russian by A. B. Sosinskil.

[21] Strictly speaking we are considering the lift of $\phi$ to the line.

[22] C. Liverani. Central limit theorem for deterministic systems. In International Conference on Dynamical Systems (Montevideo, 1995), pages 56-75. Longman, Harlow, 1996.

[23] M. Viana. Stochastic dynamics of deterministic systems. Lecture Notes XXI Braz. Math. Colloq. IMPA, Rio de Janeiro, 1997.

[24] L.-S. Young. Developments in chaotic dynamics. Notices Amer. Math. Soc., 45(10):1318-1328, 1998.

[25] K.K. Likharev. Dynamics of Josephson Junctions and Circuits. Gordon and Breach, Philadelphia, 1982.

[26] R.E. Best. Phase-Locked Loops. McGraw-Hill, New York, 1984.

[27] J.Y. Chen, K. W. Wong, H.Y. Zheng, and J.W. Shuai. Phase signal coupling induced $n: m$ phase synchronization in drive-response oscillators. Phys. Rev. E, 63:036214-1 - 036214-6, 2001.

[28] Y. Kifer. Averaging in dynamical systems and large deviations. Invent. Math., 110(2):337-370, 1992.

[29] Y. Kifer. Limit theorems in averaging for dynamical systems. Ergodic Theory Dynam. Systems, 15(6):1143-1172, 1995.

[30] J. A. Sanders and F. Verhulst. Averaging methods in nonlinear dynamical systems. Springer-Verlag, New York, 1985.

[31] An example of such a system can be found at http://math.bu.edu/people/josic/.

[32] Y. Kifer. Averaging in differnce equations driven by dynamical systems. preprint, 2001. 
[33] K. Josić and M. Palma. Phase response curves for phase coherent chaotic attractors. unpublished, 2000.

[34] J. Guckenheimer and P. Holmes. Nonlinear oscillations, dynamical systems, and bifurcations of vector fields. Springer-Verlag, New York, 1983. 